\title{
How to catch smoothing properties and analyticity of functions by computers?*
}

\author{
L.P. Castro, H. Fujiwara, T. Qian and S. Saitoh
}

\begin{abstract}
We would like to propose a new method in view to catch smoothing properties and analyticity of functions by computers. Of course, in the strict sense, such goal is impossible. However, we would like to propose some practical method that may be applied for many concrete cases for some good functions (but not for bad functions, in a sense). Therefore, this may be viewed as a procedure proposal which includes numerical experiments for the just mentioned challenge and within a new method.
\end{abstract}

\section{Introduction}

The main idea behind the present work is to answer - at least partially - to the very important practical question:

\footnotetext{
* Accepted author's manuscript (AAM) published in [L.P. Castro, H. Fujiwara, T. Qian and S. Saitoh: How to catch smoothing properties and analyticity of functions by computers?, Panos Pardalos and Themistocles M. Rassias (edts), Mathematics without Boundaries: Surveys in Interdisciplinary Research, 101-116, Springer-Verlag, New York, 2014.] [DOI: 10.1007/978-1-4939-1124-0_4] The final publication is available at Springer via http://link.springer.com/chapter/10.1007/978-1-4939-1124-0_4

L. P. Castro and S. Saitoh

CIDMA-Center for Research and Development in Mathematics and Applications, Department of Mathematics, University of Aveiro, 3810-193 Aveiro, Portugal, e-mail: castro@ua.pt; saburou.saitoh@gmail.com

H. Fujiwara

Graduate School of Informatics, Kyoto University, Kyoto, 606-8501, Japan, e-mail: fujiwara@acs.i.kyoto-u.ac.jp

T. Qian

Faculty of Science and Technology, University of Macau, Taipa, Macau, China, e-mail: fsttq@umac.mo
} 
Could we catch some smoothing properties and analyticity of functions by using computers?

More than answering affirmatively to this question, by applying the theory of reproducing kernels, we would like to propose a new method which turns possible to catch smoothing properties and analyticity of functions by computers. Clearly, in a strict mathematical sense, such methods will be impossible. Anyway, from a certain practical point of view, we would like to propose a method that may be applied for many cases where some good functions are involved, despite the fact that it will not solve other situations where "bad functions" occur. Our main arguments will be therefore be concerned with numerical experiments attaining such challenge by a new concept.

For some special cases, certain abstract theory is known by using specific approximation mathematical concepts. For example, for the analyticity issue, it is worth mentioning that our problem is closely related to some classical problems. This is the case of the problem of best analytic approximation in $L^{p}$ on the unit circle which may be formulated as follows: given a function $g \in L^{p}$, find a function $p_{g}$ in the Hardy space $H^{p}$, such that

$$
\left\|g-p_{g}\right\|_{L^{p}}=\operatorname{dist}_{L^{p}}\left(g, H^{p}\right) .
$$

This has obviously a long known history which from the point of view of powerful results may be considered to begin with the great step of F. Riezs when, in 1920, he proved [8] that the best $H^{1}$-approximation in $L^{1}$ of a trigonometric polynomial of degree $n$ is an analytic polynomial of degree at most $n$. This was even generalized in 1950 by A. Macintyre and W. Rogosinski [7], when considering the problem of best analytic approximation in $L^{p}$ for rational functions with finite number of poles in the open unit disk. In particular, it was derived that the best $H^{1}$-approximation of a rational function is also a rational function (and the same holds for the best $H^{\infty}$-approximation).

In the present paper, in order to consider analyticity or smoothing properties, we shall represent them by the members of reproducing kernel Hilbert spaces. Indeed, by the consequent Sobolev spaces, we can classify the smoothness properties by the corresponding orders in a quite useful way. On the unit disc in the complex plane, by the Bergman-Selberg spaces, we can classify the family of analytic functions, for example. In this way, many properties of analytic functions may be classified by reproducing kernel Hilbert spaces, similarly. So, our interest turns to the problem that we want to determine whether any given function belongs to a certain reproducing kernel Hilbert space or not.

\section{Preliminaries and the basic starting points}

First, we shall recall the reproducing kernel Hilbert spaces for their essences (cf. [9, 10]). 
Let $\mathscr{H}$ be a Hilbert (possibly finite-dimensional) space, and consider $E$ to be an abstract set and $\mathbf{h}$ a Hilbert $\mathscr{H}$-valued function on $E$. Then, we are able to consider the linear transform

$$
f(p)=(\mathbf{f}, \mathbf{h}(p)) \mathscr{H}, \quad \mathbf{f} \in \mathscr{H},
$$

from $\mathscr{H}$ into the linear space $\mathscr{F}(E)$ comprising all the complex valued functions on $E$. In order to investigate the linear mapping (1), we form a positive definite quadratic form function $K(p, q)$ on $E \times E$ defined by

$$
K(p, q)=(\mathbf{h}(q), \mathbf{h}(p))_{\mathscr{H}} \quad \text { on } \quad E \times E .
$$

Then, we obtain the following fundamental results.

\section{Proposition 1.}

(I) The range of the linear mapping (1) by $\mathscr{H}$ is characterized as the reproducing kernel Hilbert space $H_{K}(E)$ admitting the reproducing kernel $K(p, q)$ whose characterization is given by the two properties: $(i) K(\cdot, q) \in H_{K}(E)$ for any $q \in E$ and, (ii) for any $f \in H_{K}(E)$ and for any $p \in E,(f(\cdot), K(\cdot p))_{H_{K}(E)}=f(p)$.

(II) In general, we have the inequality

$$
\|f\|_{H_{K}(E)} \leq\|\mathbf{f}\|_{\mathscr{H}} .
$$

Here, for any member $f$ of $H_{K}(E)$ there exists a uniquely determined $\mathbf{f}^{*} \in \mathscr{H}$ satisfying

$$
f(p)=\left(\mathbf{f}^{*}, \mathbf{h}(p)\right)_{\mathscr{H}} \quad \text { on } \quad E
$$

and

$$
\|f\|_{H_{K}(E)}=\left\|\mathbf{f}^{*}\right\|_{\mathscr{H}}
$$

(III) In general, we have the inversion formula in (1) in the form

$$
f \mapsto \mathbf{f}^{*}
$$

in (II) by using the reproducing kernel Hilbert space $H_{K}(E)$.

The inversions of the type (4) are - in general - very difficult and delicate problems, see the history, for example, in [2].

The next result will exhibit that a reproducing kernel Hilbert space is a good and natural functions space:

Proposition 2. For a Hilbert space $H$ comprising of functions $\{f(p)\}$ on a set $E$, the space admits a reproducing kernel if and only if, for any point $q \in E, f \rightarrow f(q)$ is a bounded linear functional on $H$. If a function sequence $\left\{f_{n}\right\}$ converges to $f$ in the space $H$, then it converges to the function point wisely on E. Furthermore, on a subset of $E$ where $K(p, p)$ is bounded, its convergence is uniform.

We shall call a complex-valued function $k(p, q)$ in a set $E \times E$ a positive definite quadratic form function (or, a positive semi-definite matrix) on the set $E$ when it 
satisfies the property: for an arbitrary function $X(p)$ on $E$ that is zero on $E$ except for a finite number of points of $E, \sum_{p, q} \overline{X(p)} X(q) k(p, q) \geq 0$.

As we can see simply, a reproducing kernel $K(p, q)$ on $E$ is a positive definite quadratic form function on $E$, its converse statement is very important:

Proposition 3. For any positive definite quadratic form function $K(p, q)$ on $E$, there exists a uniquely determined reproducing kernel Hilbert space $H_{K}$ admitting the reproducing kernel $K(p, q)$ on $E$.

Now, for our present challenge, we shall first recall the fundamental property: For two positive semi-definite matrices $K^{(1)}(p, q)$ and $K^{(2)}(p, q)$ on $E$, if $K^{(2)}(p, q)-$ $K^{(1)}(p, q)$ is a positive semi-definite matrix on $E$, then we shall write it as follows:

$$
K^{(1)} \ll K^{(2)}
$$

We see that the symbol $\ll$ is a partial ordering in the class of all positive semidefinite matrices on $E$, and

Proposition 4. For two positive semi-definite matrices $K^{(1)}(p, q)$ and $K^{(2)}(p, q)$ on $E$,

$$
H_{K^{(1)}} \subseteq H_{K^{(2)}} \quad(\text { as members })
$$

if and only if there exists a positive constant $\Gamma$ such that

$$
K^{(1)}(p, q) \ll \Gamma^{2} K^{(2)}(p, q) .
$$

Here, the minimum of such constants $\Gamma$ coincides with the norm of the inclusion map $J$ from $H_{K^{(1)}}$ into $H_{K^{(2)}}$.

Note that for an arbitrary complex-valued function $f$ on a set $E$, the function $f(p) \overline{f(q)}$ is a positive definite quadratic form function on $E$. Therefore, we can apply the theory of reproducing kernels to the membership problem as follows:

Corollary 1. Let $K(p, q)$ be a positive definite quadratic form function on a set E. Then, for any complex-valued function $f$ on the set $E$, $f$ belongs to the reproducing kernel Hilbert space $H_{K}$ if and only if $f(p) \overline{f(q)} \ll \gamma^{2} K(p, q)$ for some constant $\gamma$. Then, the constant $\gamma$ may be taken by

$$
\|f\|_{H_{K}}=\inf \left\{\gamma>0: f(p) \overline{f(q)} \ll \gamma^{2} K(p, q)\right\} .
$$

In connection with this criteria for the membership problem, we note that for any large number of points $\left\{p_{j}\right\}_{j=1}^{n}$ of the set $E$, we cannot apply the result by the positive definiteness.

Indeed, we shall assume that, without loss of generality, $\left\{K\left(p, p_{j}\right)\right\}_{j=1}^{n}$ are linearly independent in $H_{K}$. Then, for any given values $\left\{\alpha_{j}\right\}_{j=1}^{n}$, there exists a uniquely determined member $f \in H_{K}$ satisfying

$$
f\left(p_{j}\right)=\alpha_{j}, \quad j=1,2,3, . ., n,
$$


as follows:

$$
f(p)=\sum_{j=1}^{n} C_{j} K\left(p, p_{j}\right)
$$

where the constants $\left\{C_{j}\right\}$ are determined by the equations

$$
\sum_{j=1}^{n} C_{j} K\left(p_{j^{\prime}}, p_{j}\right)=\alpha_{j^{\prime}}, \quad j^{\prime}=1,2, \ldots, n,
$$

and we obtain the inequality (5) for

$$
\gamma^{2}=\sum_{j=1}^{n} \sum_{j^{\prime}=1}^{n} C_{j} \overline{C_{j^{\prime}}} K\left(p_{j^{\prime}}, p_{j}\right)
$$

Note that the function $f$ satisfying (6) is not uniquely determined, but the function $f$ given by (7) has the minimum norm among the functions $f$ satisfying (6).

For any finite number of points $\left\{p_{j}\right\}_{j=1}^{n}$ and any given values $\left\{\alpha_{j}\right\}_{j=1}^{n}$, there exists a function $f \in H_{K}$ satisfying (6), certainly. However, for many points $\left\{p_{j}\right\}_{j=1}^{n}$ and bad values $\left\{\alpha_{j}\right\}_{j=1}^{n}$, the calculations (7) to looking for $\left\{C_{j}\right\}$ will be numerically and practically difficult. The difficulty to calculate (7) will depend on the given data and the function space $H_{K}$. We looked such phenomena for the Paley-Wiener spaces in some cases (cf. [1]). However, to represent such deep and delicate phenomena exactly will be difficult. Anyway, we may expect that the smoothness property of functions may be reflected to some properties on a large point set. We shall propose such method in the next section in view to understand how to catch such property more clearly.

The goodness of a function in the reproducing kernel $H_{K}$ may be given by:

(g1) the number of the points $\left\{p_{j}\right\}$ in (7)

(g2) the distribution of the coefficients $\left\{C_{j}\right\}$ in (7), and

(g3) the distribution of the points $\left\{p_{j}\right\}$ on the set $E$.

The factors (g1) and (g2) may be considered in a general setting, however, (g3) will depend on the reproducing kernel Hilbert space $H_{K}$.

\section{Aveiro discretization method}

By considering the inversion of (1) from a finite number of point data $\left\{f\left(p_{j}\right)\right\}$, we established the following fundamental results:

Proposition 5 (Ultimate realization of reproducing kernel Hilbert spaces ([2])). In our general situation and for a uniqueness set $\left\{p_{j}\right\}$ of the set $E$ for the function space $H_{K}$ (that is, for any function $f \in H_{K}$ satisfying $f\left(p_{j}\right)=0$ for all $j$, $f$ is zero identically on $E$ ) satisfying the linearly independence in $H_{K}$, we obtain, for any member $f \in H_{K}$ 


$$
\|f\|_{H_{K}}^{2}=\lim _{n \rightarrow \infty} \sum_{j=1}^{n} \sum_{j^{\prime}=1}^{n} f\left(p_{j}\right) \widetilde{a_{j j^{\prime}}} \overline{f\left(p_{j^{\prime}}\right)},
$$

where $\widetilde{a_{j j^{\prime}}}$ are assumed the elements of the complex conjugate inverse of the positive definite Hermitian matrix $A_{n}$ constituted by the elements

$$
a_{j j^{\prime}}=K\left(p_{j^{\prime}}, p_{j}\right) .
$$

Furthermore, the limit is determined in the sense of not decreasing sequences.

From this Proposition 5, we see that the membership property is faithfully reflected on the uniqueness point set $\left\{p_{j}\right\}$ by the norm (10). Now, our basic idea is as follows: Fujiwara's infinite precision algorithm and great computer power will be able to calculate the norm (10) for many practical cases; see the case of numerical and real inversion formula of the Laplace transform that is a famous difficult problem (cf. $[1,2,4,5,6])$. Surprisingly enough, Fujiwara gave the solution for some Fredholm integral equation containing a parameter $\alpha=10^{-400}$ by 6000 discretization (6000 linear equations) with $\mathbf{6 0 0}$ digits precision.

Furthermore, Fujiwara gave already numerical experiments for the norms for many points (cf. [1,2]), and we see that when increasing the number of points we are requested to calculate with more precision and more calculation costs. So, we will need more precision and computer costs for the realization of our method. However, this was succeeded already for our present method as we shall see in Section 5.

\section{The Sobolev spaces and the Paley-Wiener spaces}

In order to give numerical experiments, we shall introduce the typical reproducing kernel Hilbert spaces, Sobolev Hilbert spaces.

Let $m>\frac{n}{2}$ be an integer. Denote by ${ }_{N} C_{K}$ the binomial coefficient. Then, we have

$$
W^{m, 2}\left(\mathbb{R}^{n}\right)=H_{K}\left(\mathbb{R}^{n}\right),
$$

where $W^{m, 2}\left(\mathbb{R}^{n}\right)$ denotes the Sobolev space whose norm is given by

$$
\|F\|_{W^{m, 2}\left(\mathbb{R}^{n}\right)}=\sqrt{\sum_{v=0}^{m}{ }_{m} C_{V}\left(\sum_{\alpha \in \mathbb{Z}_{+}^{n},|\alpha| \leq v}^{v} \frac{v !}{\alpha !} \int_{\mathbb{R}}\left|\frac{\partial^{v} F(x)}{\partial x^{v}}\right|^{2} d x\right)}
$$

and

$$
K(x, y)=\frac{1}{(2 \pi)^{n}} \int_{\mathbb{R}} \frac{\exp (i(x-y) \cdot \xi)}{\left(1+|\xi|^{2}\right)^{m}} d \xi
$$

In particular, note that if $m>\frac{n}{2}$, then $W^{m, 2}\left(\mathbb{R}^{n}\right)$ is embedded into $\mathrm{BC}\left(\mathbb{R}^{n}\right)$. 
A generalization of the above spaces is given in the following way. Let $s>\frac{n}{2}$ and define

$$
K(x, y):=\frac{1}{(2 \pi)^{n}} \int_{\mathbb{R}^{n}}\left(1+|\xi|^{2}\right)^{-s} \exp (i(x-y) \cdot \xi) d \xi .
$$

Then, we have

$$
H_{K}\left(\mathbb{R}^{n}\right)=H^{s}\left(\mathbb{R}^{n}\right)
$$

where the norm is given by

$$
\|f\|_{H^{s}\left(\mathbb{R}^{n}\right)}=\left(\int_{\mathbb{R}^{n}}\left(1+|\xi|^{2}\right)^{s}|\mathscr{F} f(\xi)|^{2} d \xi\right)^{\frac{1}{2}} .
$$

The simplest example is given in the following way. The space $H_{S}(\mathbb{R})$ is made up of absolutely continuous functions $F$ on $\mathbb{R}$ with the norm

$$
\|F\|_{H_{S}(\mathbb{R})}:=\sqrt{\int_{\mathbb{R}}\left(F(x)^{2}+F^{\prime}(x)^{2}\right) d x} .
$$

In addition, the Hilbert space $H_{S}(\mathbb{R})$ admits the reproducing kernel

$$
K_{1}(x, y):=\frac{1}{2 \pi} \int_{\mathbb{R}} \frac{1}{1+\xi^{2}} \exp (i(x-y) \xi) d \xi=\frac{1}{2} e^{-|x-y|} .
$$

Note that if the factor $\left(1+|\xi|^{2}\right)^{s}$ is replaced by the characteristic function on a compact set on $\mathbb{R}^{n}$, then that space becomes the Paley-Wiener space comprising of entire functions of exponential type.

Indeed, we shall consider the following integral transform, for a function $F$ in

$$
\begin{aligned}
& L_{2}(-\pi / h,+\pi / h), \quad h>0: \\
& f(z)=\frac{1}{2 \pi} \int_{-\pi / h}^{\pi / h} F(t) e^{-i z t} d t .
\end{aligned}
$$

In order to identify the image space following the theory of reproducing kernels, we form the reproducing kernel

$$
K_{h}(z, \bar{u})=\frac{1}{2 \pi} \int_{-\pi / h}^{\pi / h} e^{-i z t} \overline{e^{-i u t}} d t=\frac{1}{\pi(z-\bar{u})} \sin \frac{\pi}{h}(z-\bar{u}) .
$$

The corresponding image space is called the Paley-Wiener space $W\left(\frac{\pi}{h}\right)$, comprised of all analytic functions of exponential type satisfying, for some constant $C$, and as $z \rightarrow \infty$,

$$
|f(z)| \leq C \exp \left(\frac{\pi|z|}{h}\right)
$$

and

$$
\int_{\mathbf{R}}|f(x)|^{2} d x<\infty
$$


From the identity

$$
K_{h}\left(j h, j^{\prime} h\right)=\frac{1}{h} \delta\left(j, j^{\prime}\right)
$$

(where we are using the Kronecker's $\delta$ ), since $\delta\left(j, j^{\prime}\right)$ is the reproducing kernel for the Hilbert space $\ell^{2}$, using the general theory of integral transforms of Proposition 1 and the Parseval's identity, we reach to the isometric identities in (19)

$$
\frac{1}{2 \pi} \int_{-\pi / h}^{\pi / h}|F(t)|^{2} d t=h \sum_{j}|f(j h)|^{2}=\int_{\mathbb{R}}|f(x)|^{2} d x .
$$

That is, the reproducing kernel Hilbert space $H_{K_{h}}$ with $K_{h}(z, \bar{u})$ is characterized as a space comprising the Paley-Wiener space $W\left(\frac{\pi}{h}\right)$ and with the square norm above. Here we used the well-known result that $\{j h\}_{j}$ is a uniqueness set for the PaleyWiener space $W\left(\frac{\pi}{h}\right)$; that is, $f(j h)=0$ for all $j$ implies $f \equiv 0$. Then, the reproducing property of $K_{h}(z, \bar{u})$ states that

$$
f(x)=\left(f(\cdot), K_{h}(\cdot, x)\right)_{H_{K_{h}}}=h \sum_{j} f(j h) K_{h}(j h, x)=\int_{\mathbb{R}} f(\xi) K_{h}(\xi, x) d \xi .
$$

In particular, on the real line $x$, this representation is the sampling theorem which represents the whole data $f(x)$ in terms of the discrete data $\{f(j h)\}_{j}$. For a general theory for the sampling theory and error estimates for some finite points $\{h j\}_{j}$, see [9].

\section{Numerical experiments}

In order to look our principle, we show some numerical examples. In our numerical experiments, we take $n$ sampling points $p_{j}, j=1,2, \ldots, n$, and calculate a truncation of the right-hand side of (10):

$$
\sum_{j, j^{\prime}=1}^{n} f\left(p_{j}\right) \widetilde{a_{j j^{\prime}}} \overline{f\left(p_{j^{\prime}}\right)}
$$

which converges to the square of $H_{K}$-norm of $f$ if the test function $f$ belongs to the target function space $H_{K}$. The sampling points are distributed in some finite interval uniformly by using pseudo-random numbers generated by the standard library function rand ( ) in the programming language C. Throughout this section, we use 300 decimal digits arithmetic by the multiple-precision arithmetic environment exflib[3].

Example 1. Does $f_{1}(x)=\frac{1}{2} e^{-|x|}$ belong to $H_{S}(\mathbb{R})$ whose reproducing kernel is given by (18)?

The Figure 1 shows our numerical results. The interval where the sampling points are located is set to be $(-5,5)$. The horizontal axis is the number of sampling points 


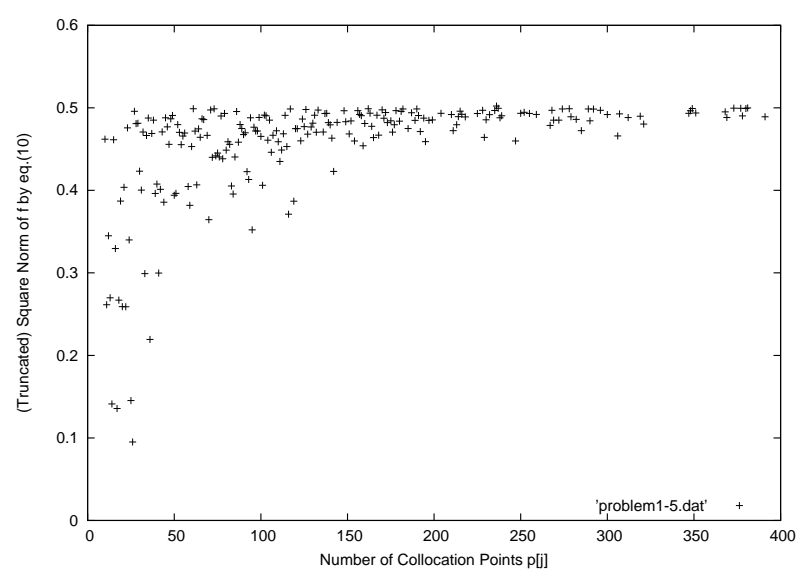

Fig. 1 Numerical Results for Example 1, $p_{j} \in(-5,5)$.

$n$, and the vertical axis is the approximated norm (21). The graph shows its convergence as $n$ becomes large, and it means that $f_{1}$ belongs to $H_{S}(\mathbb{R})$.

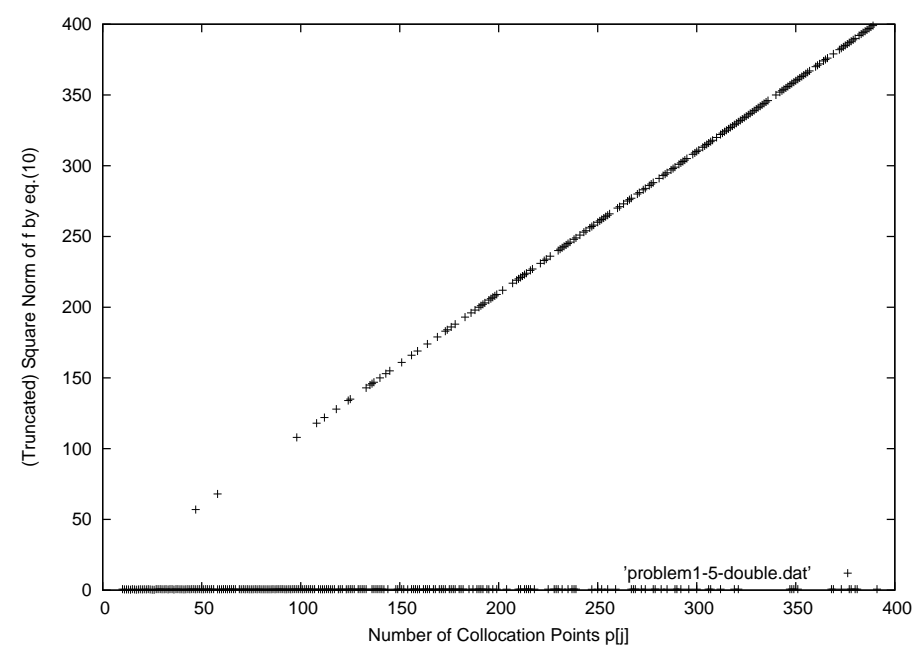

Fig. 2 Numerical Results for Example 1 with Double Precision Arithmetic

Next, we process the same procedure with the standard double precision arithmetic. The result shown in Figure 2 is quite different from Figure 1, and the differences are due to accumulation of rounding errors in floating-point arithmetic. From the results we know that multiple-precision arithmetic is essentially required for the proposed algorithm. 


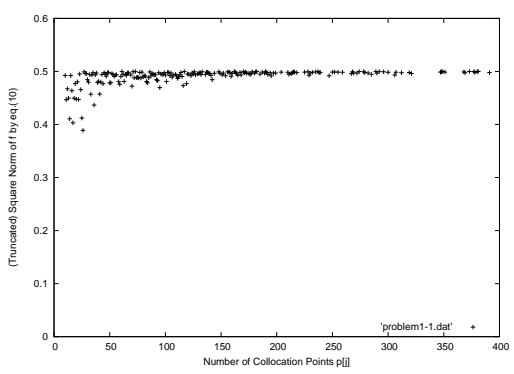

(a) $p_{j} \in(-1,1)$

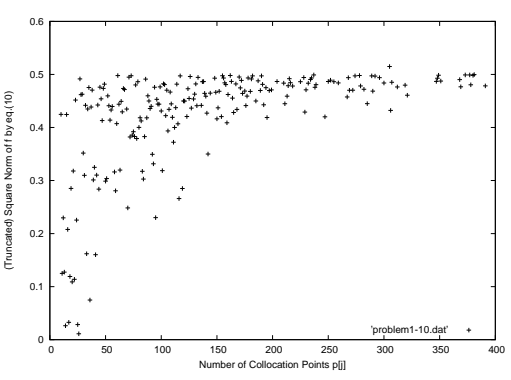

(b) $p_{j} \in(-10,10)$

Fig. 3 Numerical Results for Example 1 with Different Intervals

Figure 3 shows results with different intervals. Comparing Figure 1 with Figure 3 , it can be seen that larger intervals return larger dispersion in this example.

Example 2. We check that $f_{2}(x)=\frac{1}{4} e^{-|x|}(1+|x|)$ belongs to $H_{2}(\mathbb{R})$ whose reproducing kernel is given by

$$
K(x, y)=\frac{1}{4} e^{-|x-y|}(1+|x-y|) .
$$

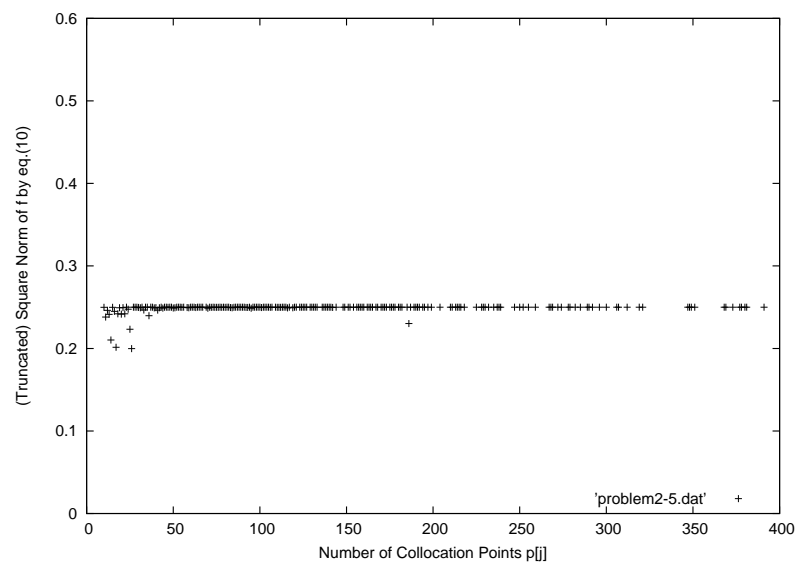

Fig. 4 Numerical Results for Example 2, $p_{j} \in(-5,5)$.

Figure 4 shows numerical results, where the computed values show pretty quick convergence. It obviously indicates that $f_{2}$ is a member of $H_{2}(\mathbb{R})$.

Example 3. Is $f_{1}(x)=\frac{1}{2} e^{-|x|}$ a member of $H_{2}(\mathbb{R})$ ? 


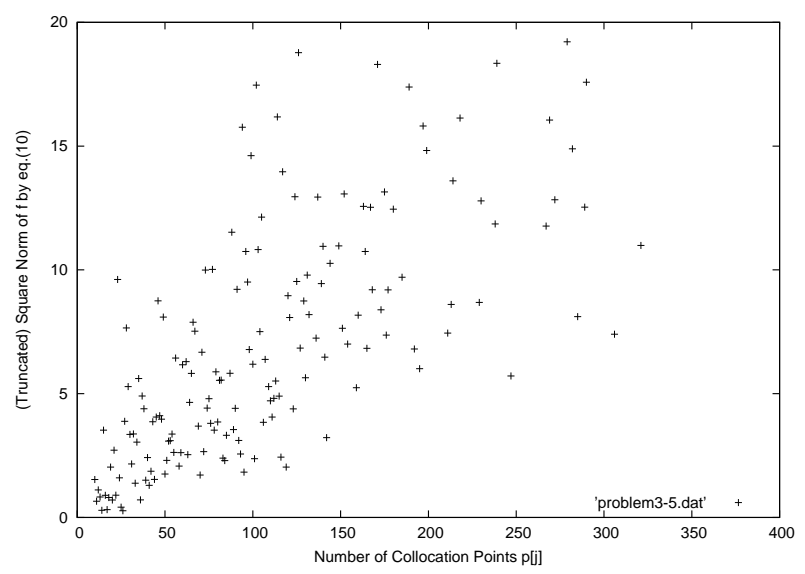

Fig. 5 Numerical Results for Example 3, $p_{j} \in(-5,5)$.

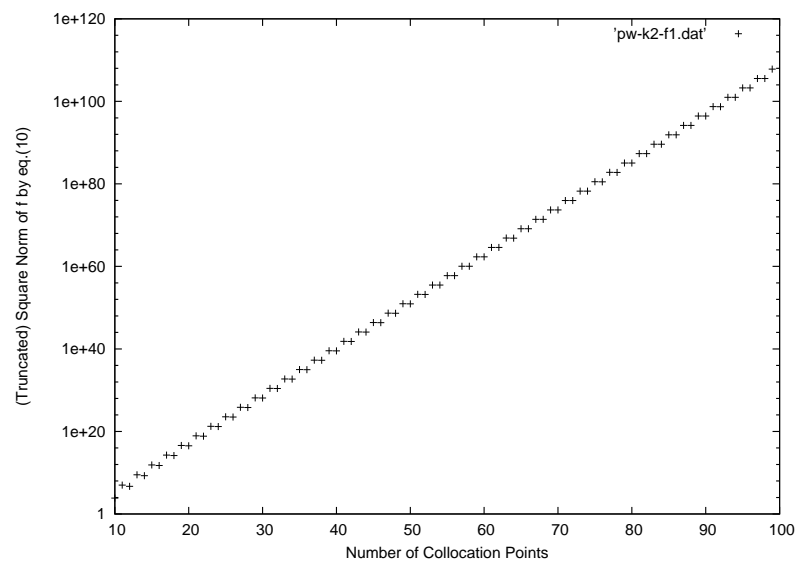

Fig. 6 Numerical Results for Example 4 with $h=1$.

Our numerical results are shown in Figure 5. We note that the vertical scale is different from that in Figure 1 or Figure 4. We cannot see convergence in numerical results. In other words, it suggests that the function $f_{1}$ does not belong to $H_{2}(\mathbb{R})$.

Example 4. We consider situations of Paley-Wiener spaces whose reproducing kernel is given by (20).

In the following examples, we distribute collocation points $p_{j}$ in the interval $(-5,5)$, uniformly, and use 300 decimal digits computation.

We denote

$$
g_{h}(z)=K_{h}(z, 0)
$$




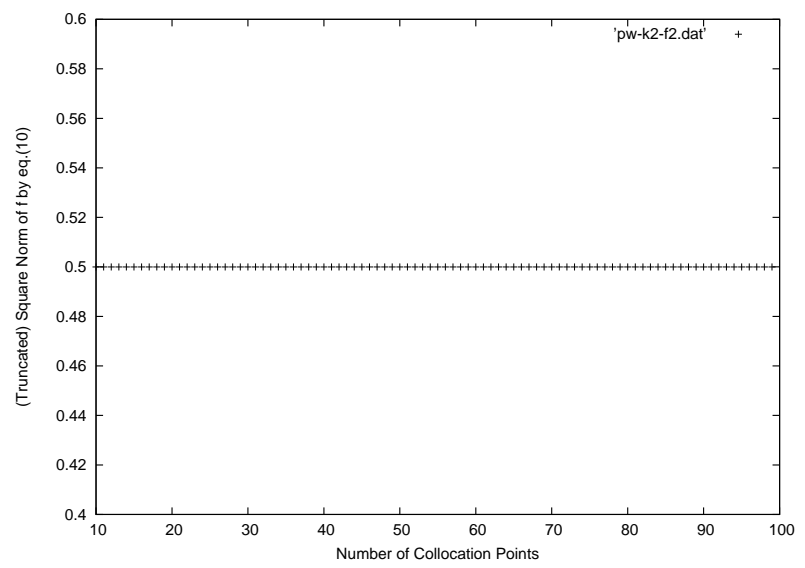

Fig. 7 Numerical Results for Example 4 with $h=2$.

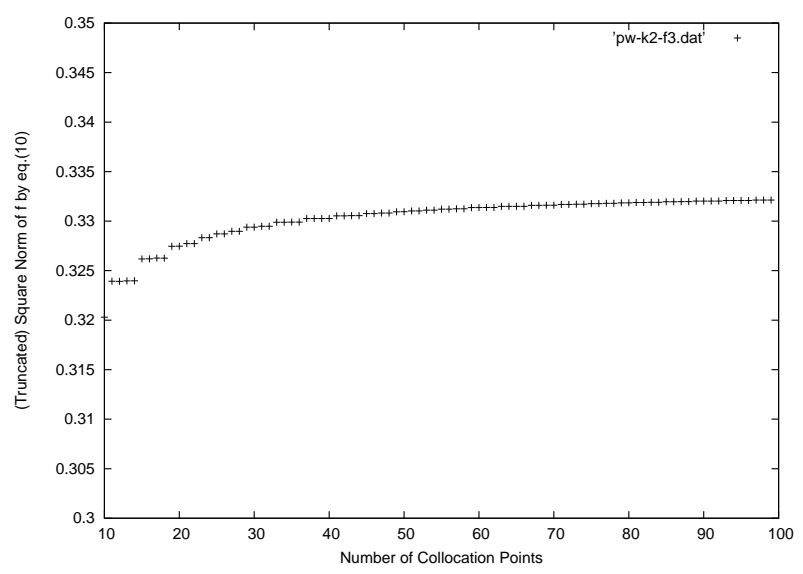

Fig. 8 Numerical Results for Example 4 with $h=3$.

and check whether $g_{h}$ belongs to the space $W\left(\frac{\pi}{2}\right)$.

Figures 6, 7 and 8 show our numerical results for $g_{1}, g_{2}$ and $g_{3}$, respectively. Figure 6 , where the norm grows exponentially, indicates that $g_{1} \notin W\left(\frac{\pi}{2}\right)$. On the other hand, in Figures 7 and 8, the computed norms illustrate convergence, indicating in this way that both $g_{2}$ and $g_{3}$ belong to $W\left(\frac{\pi}{2}\right)$.

Finally, we check whether $f_{1}$ and $f_{2}$ in Example 1 and 2 belong to $W\left(\frac{\pi}{2}\right)$. Our results are shown in Figures 9, and they imply that both $f_{1}$ and $f_{2}$ do not belong to $W\left(\frac{\pi}{2}\right)$. 


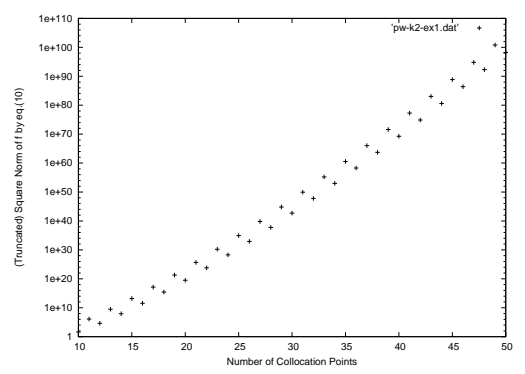

(a) Testing $f_{1} \in W(\pi / 2)$

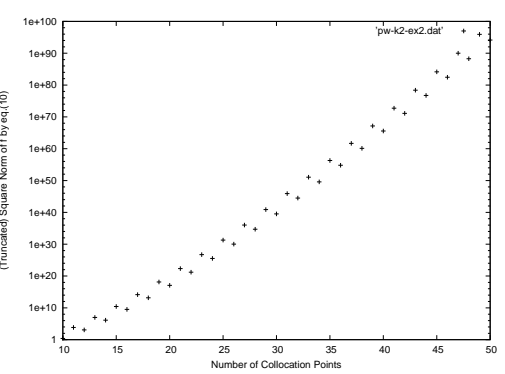

(b) Testing $f_{2} \in W(\pi / 2)$

Fig. 9 Numerical Results for Example 4

\section{Band preserving, phase retrieval and related problems}

The membership problem that we are considering here was strongly and directly motivated by the deep analytical results in [11]. The corresponding fundamental problems, with many applications to analytical signals, are stated in our general theory as follows: For any fixed member $f \in H_{K}$, look for a function $g$ satisfying

$$
f(p) g(p) \in H_{K}
$$

Indeed, they examined deeply for the special Paley-Wiener space for the images by Fourier inversion for the $L_{2}$ functions on an interval $[0, A]$.

If we are concerned with the functions $f$ and $g$ on a finite number of points $\left\{p_{j}\right\}$, then for the non-vanishing points $\left\{p_{j}\right\}$ of the function $f$, the values $g\left(p_{j}\right)$ are given arbitrary, and we obtain the representation

$$
f(p) g(p)=\sum_{j=1}^{n} C_{j} K\left(p, p_{j}\right),
$$

as in (7) and we can obtain the meromorphic function $g$ completely, because the function $f$ and the right-hand side are analytic functions. We can assume, without loss of generality, that the functions $\left\{K\left(p, p_{j}\right)\right\}$ are linearly independent and in this case, the function (23) is uniquely determined.

The above logic is very interesting, because in view to look for the functions $g$, we can see a great freedom. Furthermore, we note that, indeed, for the function $g$, we can take the functions:

$$
g(p)=\frac{h(p)}{f(p)} \quad\left(\text { for any given function } h \in H_{K}\right),
$$

which are meromorphic functions. For the membership problem, we can conclude a simple result. So, we shall recall the reference [11] and we note that all the logics in the paper depend on the property of the restriction for the functions $g$ that belong 
to $L_{p}$ spaces on the real line. We will not be able to consider in a simpler manner such condition on $g$ in the above idea. So, we wonder if in future it will be possible to expose some connection between the above theory and the very hard analysis obtained in [11].

Moreover, it is also significant to notice that all the analytical and deep theory of [11] depends on the zero point properties of the function $f$, of course. So, consequently, the construction of the desired functions $g$ is not simple. When we consider the support properties of the related Fourier inversions in connection with the convolution property, the results will be mysteriously deep and many concrete problems may happen. For the sake of hard analysis, the authors of [11] were able to solve surprisingly and perfectly the phase retrieval problem which is requesting, however, the strong condition $|g|=1$ on the real line. As it was exposed above, for the sake of great freedom for the functions $g$, we can construct the desired function $g$ even in the case of phase retrieval problems.

Acknowledgements This work was supported in part by Portuguese funds through the CIDMACenter for Research and Development in Mathematics and Applications, and the Portuguese Foundation for Science and Technology (FCT), within project PEst-OE/MAT/UI4106/2014. The fourth named author is supported in part by the Grant-in-Aid for the Scientific Research (C) (2) (No. 24540113).

\section{References}

1. L. P. Castro, H. Fujiwara, M. M. Rodrigues and S. Saitoh, A new discretization method by means of reproducing kernels, in: Interactions between Real and Complex Analysis, Le Hung Son and Wolgang Tutscheke (edts.), Sci. and Tech. Publication House, Hanoi, 2012, 185-223.

2. L.P. Castro, H. Fujiwara, M.M. Rodrigues, S. Saitoh and V. K. Tuan, Aveiro Discretization Method in Mathematics: A New Discretization Principle, in: Mathematics without Boundaries: Surveys in Pure Mathematics, Panos Pardalos and Themistocles M. Rassias (edts.), Springer-Verlag, New York, 52 pp., to appear.

3. H. Fujiwara, exflib - multiple-precision arithmetic library, http://www-an.acs.i.kyoto-u.ac.jp/ fujiwara.

4. H. Fujiwara, Applications of reproducing kernel spaces to real inversions of the Laplace transform, RIMS Koukyuuroku 1618 (2008), 188-209.

5. H. Fujiwara, T. Matsuura, S. Saitoh and Y. Sawano, Numerical real inversion of the Laplace transform by using a high-accuracy numerical method, Further Progress in Analysis, World Sci. Publ., Hackensack, NJ, 2009, 574-583.

6. H. Fujiwara, Numerical real inversion of the Laplace transform by reproducing kernel and multiple-precision arithmetric, Progress in Analysis and its Applications, Proceedings of the 7th International ISAAC Congress, World Scientific, 2010, 289-295.

7. A. J. Macintyre and W. W. Rogosinski, Extremum problems in the theory of analytic functions, Acta Math. 82 (1950), 275-325.

8. F. Riesz, Über Potenzreihen mit vorgeschriebenen Anfangsgliedern, Acta Math. 42 (1920), 145-171.

9. S. Saitoh, Integral Transforms, Reproducing Kernels and their Applications, Pitman Res. Notes in Math. Series 369, Addison Wesley Longman, Harlow, 1997. 
10. S. Saitoh, Theory of reproducing kernels: Applications to approximate solutions of bounded linear operator functions on Hilbert spaces, Amer. Math. Soc. Transl. Ser. 2, 230, Amer. Math. Soc., Providence, RI, 2010.

11. L. Tan and T. Qian, Backward shift invariant subspaces with applications to band preserving and phase retrieval problems (manuscript). 